\title{
Role of cerebro-placental ratio in prediction of perinatal outcome in high-risk pregnancies with intrauterine growth restriction
}

\author{
Original Mohamed Ahmed Hassan El-Kady ${ }^{l}$, Esraa Hamdy Mohammed Mohammed ${ }^{1,2}$, Ebtihal \\ Article Mohamed Eltaieb1 \\ ${ }^{1}$ Department of Obstetrics and Gynecology, Faculty of Medicine, Ain Shams University, Cairo \\ and ${ }^{2}$ Department of Obstetrics and Gynecology, El Salam Specialized Hospital, Cairo
}

\begin{abstract}
Introduction: Doppler ultrasound velocimetry of uteroplacental umbilical and fetal vessels has become established method of antenatal monitoring. Cerebroplacental ratio has been studied to predict neonatal outcomes.

Aim of the work: Our aim was to assess role of cerebro-placental ratio in prediction of perinatal outcome in high-risk pregnancies with intrauterine growth restriction.

Patients and Methods: In this study was held in Ain Shams university maternity hospital which included 60 high risk pregnant women divided in to two groups: Group I: study group: 30 high risk pregnant women with IUGR, Group IIcontrol group: 30 high risk pregnant women without IUGR.

Results: The current study showed that among patients with IUGR and low CPR, $17(56.7 \%)$ had CS for fetal distress, 12 $(40.0 \%)$ had neonate with low APGAR, $13(43.3 \%)$ had neonatal meconium aspiration, 6 (20.0\%) had neonatal Hypoxic ischemic Encephalopathy, 15 (50.0\%) had neonate need NICU admission, 3 (10.0\%) had neonatal death, 1 (3.3\%) had neonatal still birth. The current study showed that among patients without IUGR and low CPR, $8(26.7 \%)$ had CS for fetal distress, 5 (16.7\%) had neonate with low APGAR, 4 (13.3\%) had neonatal meconium aspiration, 1 (3.3\%) had neonatal Hypoxic ischemic Encephalopathy, 5 (16.7\%) had neonate need NICU admission, 1 (3.3\%) had neonatal death, $0(0.0 \%)$ had neonatal still birth.

Conclusion: Cerebro -placental ratio has high predictive value of perinatal outcome in high risk pregnancy with intrauterine growth restriction.
\end{abstract}

Key Words: Cerebro-placental ratio, high-risk pregnancies, intrauterine growth restriction, perinatal outcome.

Received: 24 January 2020, Accepted: 13 April 2020

Corresponding Author: Esraa Hamdy Mohammed, Department of Obstetrics and Gynecology, Faculty of Medicine, Ain-Shams University, Cairo, Egypt, Tel.: 01140980602, E-mail: esraahamdyebw@gmail.com.

ISSN: 2090-7265, May 2020, Vol.10, No.2

\section{INTRODUCTION}

Intrauterine growth restriction (IUGR) is defined as sonographic estimated fetal weight below 10th percentile for gestational age ${ }^{[21]}$. According to the American College of Obstetricians and Gynecologists, IUGR is one the most common and complex problems in modern obstetrics ${ }^{[2]}$.

This characterization is understandable considering the various published definitions, poor detection rate, limited preventive or treatment options, multiple associated morbidities, and increased likelihood of perinatal mortality associated with IUGR. Suboptimal growth at birth is linked with impaired intellectual performance and diseases such as hypertension and obesity in adulthood ${ }^{[6]}$. Current challenges in the clinical management of IUGR include accurate diagnosis of the truly growthrestricted fetus, selection of appropriate fetal surveillance, and optimizing the timing of delivery. Despite the potential for a complicated course, antenatal detection of IUGR and its antepartum surveillance can improve outcomes ${ }^{[12]}$.
It is important to synthesize and assess the strength of evidence of the current literature regarding the use of Doppler velocimetry of the umbilical artery and middle cerebral artery for non-anomalous fetuses with suspected IUGR, and to provide recommendations regarding antepartum management of these pregnancies, in particular for singleton gestations ${ }^{[3]}$. It has been acknowledged that defining small for gestational age (birthweight below 10th percentile for gestational age) by general population charts vs customized charts is an important issue, but this is not the focus of this clinical opinion ${ }^{[10]}$.

Intrauterine growth restriction (IUGR) is an indicator of the increased risk of perinatal and long-term mortality and morbidity when compared to those born with normal growth. There is a considerable difference in the incidence of IUGR across different populations. In babies born with a birth weight less than $2500 \mathrm{gms}$, its prevalence is almost $33 \%$. The incidence of IUGR shows a dependence on economic growth too, with a relatively lower incidence in developed countries (4-8\%) as compared to that in 
developing countries $(6 \%-30 \%)^{[5]}$.

The average incidence of IUGR is nearly $8 \%$ in the general population. In nearly $35 \%-40 \%$ of the cases, IUGR is the consequence of an abnormal condition. Factors like placental insufficiency, maternal hypertension, cardiovascular disease, diabetes, infections, low socioeconomic status, previous history and preeclampsia are some of the known risk factors for IUGR ${ }^{[1]}$.

The maintenance of good utero-placental circulation is necessary to continue a normal pregnancy. The progression of pregnancy is marked by a number of changes and adaptations in the maternal, placental and fetal vasculatures $^{[23]}$. An inability to adapt to these changes results in the development of abnormal vascular resistance patterns, which might consecutively lead to the compromise of fetal well-being and ultimately IUGR ${ }^{[19]}$. Early identification and prediction of IUGR, to a great extent, rests in an ability to evaluate the maternal, placental and fetal vascular patterns effectively and efficiently.

A number of indices based on color Doppler flowmetry have been proposed to evaluate the risk of intrauterine growth restriction in an ongoing pregnancy, some of these include the pulsatility and resistive indices (PI and RI) of the umbilical artery (UA) and that of the middle cerebral artery (MCA) in predicting adverse perinatal outcome ${ }^{[1]}$.

In the present study, an attempt will be made to evaluate the efficacy of these Doppler indices in our settings in third trimester pregnancies.

\section{AIM OF THE STUDY}

The aim of the study is to assess role of cerebroplacental ratio in prediction of perinatal outcome in highrisk pregnancies with intrauterine growth restriction.

\section{PATIENTS AND METHODS}

This case control study that was held in Ain Shams university maternity hospital, during the period between March 2019 to October 2019. The aim was to evaluate the role of cerebro-placental ratio in predictability of perinatal outcome in high-risk category of pregnancies having intrauterine growth restriction. This research recruited 60 high risk pregnant women categorized in to two research groups: Group I: study group: 30 high risk pregnant women with IUGR, Group II: control group: 30 high risk pregnant women without IUGR. With inclusion criteria of study group are: maternal age more than 20 years old, singleton Pregnant women, third trimester gestational age, high risk pregnancy as pregnancy-induced hypertension (PIH), sure of last menstrual period (LMP) date, IUGR diagnosis by sonographic estimated fetal weight below 10th percentile for gestational age. And inclusion criteria of control group are: maternal age more than 20 years old, singleton pregnant women, third trimester gestational age, high risk pregnancy as pregnancy-induced hypertension (PIH), sure of last menstrual period (LMP) date, no IUGR diagnosis by sonographic estimated fetal weight for gestational age. While exclusion criteria for both group are :gestational diabetes mellitus, congenital malformation in the present fetus, multiple fetal pregnancy, wrong LMP date. All participants in the study will receive both oral and written informed consent after explaining the details of the study for them, as agreed upon by the ethical committee. Gestational age determination was based on the best estimate from the last menstrual history and by ultrasonography (USG) or routine fetal biometry in the first trimester or early second trimester.All participants then will have ultrasound Doppler study of the following arteries: Umbilical artery, Middle cerebral artery (MCA), were examined by Color Doppler ultrasound and Pulsed wave Doppler, All Doppler examinations were performed using Medison R5 Ultrasound machine equipped with a 3.5 MHz Convex probe. Doppler indices were calculated by the built-in software programs in the machine.

\section{Statistical methods}

The collected data were coded, tabulated, and statistically analyzed using IBM SPSS statistics (Statistical Package for Social Sciences) software version 18.0, IBM Corp., Chicago, USA, 2009. The level of significance was taken at $P$ value $<0.050$ is significant, otherwise is nonsignificant.

\section{RESULTS}

Table 1 shows that no significant differences between the studied groups regarding demographic characteristics.

Table 2 shows that poor outcomes were more frequent among IUGR group; the differences were statistically signfciant except Hypoxic ischemic encephalopathy, death and still birth.

Table 3 shows that there was significant moderate agreement between low CPR and CS for fetal distress.

Table 4 shows that there was significant moderate agreement between low CPR and low APGAR.

Table 5 shows that there was significant moderate agreement between low CPR and meconium aspiration.

Table 6 shows that there was nonsignificant low agreement between low CPR and hypoxic ischemic encephalopathy. Table 7 shows that there was significant moderate agreement between low CPR and NICU admission.

Table 8 shows that There was non-significant low agreement between low CPR and neonatal death in study groups. 
Table 1: Comparison between the studied groups regarding demographic characteristics

\begin{tabular}{|c|c|c|c|c|}
\hline Variables & & $\begin{array}{c}\text { IUGR } \\
(\mathrm{N}=30)\end{array}$ & $\begin{array}{c}\text { Non-IUGR } \\
\quad(\mathrm{N}=30)\end{array}$ & $P$ \\
\hline \multirow{2}{*}{ Age (years) } & Mean \pm SD & $28.8 \pm 3.0$ & $29.2 \pm 2.8$ & \multirow{2}{*}{$0.629^{\wedge}$} \\
\hline & Range & $24.0-34.0$ & $24.0-34.0$ & \\
\hline \multirow{2}{*}{ BMI (kg/m2) } & Mean \pm SD & $27.2 \pm 2.4$ & $26.3 \pm 2.2$ & \multirow{2}{*}{$0.629^{\wedge}$} \\
\hline & Range & $22.7-32.0$ & $22.6-31.2$ & \\
\hline \multirow{2}{*}{ Parity } & Primi & $21(70.0 \%)$ & $23(76.7 \%)$ & \multirow{2}{*}{$0.559 \#$} \\
\hline & Multi & $9(30.0 \%)$ & $7(23.3 \%)$ & \\
\hline \multirow{2}{*}{ GA (weeks) } & Mean \pm SD & $35.7 \pm 1.5$ & $35.9 \pm 1.0$ & \multirow{2}{*}{$0.611^{\wedge}$} \\
\hline & Range & $34.0-38.0$ & $34.0-38.0$ & \\
\hline
\end{tabular}

^Independent t-test. \#Chi square test

Table 2: Comparison between the studied groups regarding perinatal outcomes

\begin{tabular}{|c|c|c|c|c|}
\hline Outcomes & $\begin{array}{c}\text { IUGR } \\
(\mathrm{N}=30)\end{array}$ & $\begin{array}{c}\text { Non-IUGR } \\
\quad(\mathrm{N}=30)\end{array}$ & $P$ & $\begin{array}{c}\text { OR } \\
(95 \% \mathrm{CI})\end{array}$ \\
\hline CS for fetal distress & $19(63.3 \%)$ & $11(36.7 \%)$ & \#0.039* & $2.98(1.04-8.53)$ \\
\hline Low APGAR & $14(46.7 \%)$ & $6(20.0 \%)$ & $\# 0.028 *$ & $3.50(1.11-11.02)$ \\
\hline Meconium aspiration & $13(43.3 \%)$ & $5(16.7 \%)$ & $\# 0.024 *$ & $3.82(1.15-12.71)$ \\
\hline Hypoxic ischemic encephalopathy & $6(20.0 \%)$ & $1(3.3 \%)$ & $\S 0.103$ & $7.25(0.82-64.46)$ \\
\hline NICU & $16(53.3 \%)$ & $7(23.3 \%)$ & $\# 0.017 *$ & $3.76(1.24-11.38)$ \\
\hline Death & $3(10.0 \%)$ & $1(3.3 \%)$ & $\S 0.612$ & $3.22(0.32-32.89)$ \\
\hline Still birth & $1(3.3 \%)$ & $0(0.0 \%)$ & $\S 1.000$ & -- \\
\hline
\end{tabular}

\#Chi square test. §Fisher's Exact test. *Significant

Table 3: Agreement between low CPR and CS for fetal distress

\begin{tabular}{|c|c|c|c|c|}
\hline \multicolumn{5}{|c|}{ IUGR $(\mathrm{N}=30)$} \\
\hline \multirow{2}{*}{ CPR } & \multicolumn{2}{|c|}{ CS for fetal distress } & \multirow{2}{*}{\multicolumn{2}{|c|}{ Total }} \\
\hline & Present & Absent & & \\
\hline Low & $17(56.7 \%) \mathrm{TP}$ & $3(10.0 \%) \mathrm{FP}$ & \multicolumn{2}{|c|}{$20(66.7 .0 \%)$} \\
\hline Normal & $2(6.7 \%) \mathrm{FN}$ & $8(26.7 \%) \mathrm{TN}$ & \multicolumn{2}{|c|}{$10(33.3 \%)$} \\
\hline Total & $19(63.3 \%)$ & $11(36.7 \%)$ & \multicolumn{2}{|c|}{$30(100.0 \%)$} \\
\hline Kappa $(95 \%$ CI $)$ & \multicolumn{2}{|c|}{$0.634(0.344-0.924)$} & $P$ & $<0.001^{*}$ \\
\hline \multicolumn{5}{|c|}{ Non-IUGR $(\mathrm{N}=30)$} \\
\hline Low & $8(26.7 \%) \mathrm{TP}$ & $3(10.0 \%) \mathrm{FP}$ & \multicolumn{2}{|c|}{$11(36.7 \%)$} \\
\hline Normal & $3(10.0 \%) \mathrm{FN}$ & $16(53.3 \%) \mathrm{TN}$ & \multicolumn{2}{|c|}{$19(63.3 \%)$} \\
\hline Total & $11(36.7 \%)$ & $19(63.3 \%)$ & \multicolumn{2}{|c|}{$30(100.0 \%)$} \\
\hline Kappa $(95 \%$ CI $)$ & \multicolumn{2}{|c|}{$0.569(0.264-0.875)$} & $P$ & $<0.001^{*}$ \\
\hline
\end{tabular}

Percentages are from the total (30), TP: True positive, TN: True negative, FP: False positive, FN: False negative 
Table 4: Agreement between low CPR and low APGAR

\begin{tabular}{|c|c|c|c|c|}
\hline \multicolumn{5}{|c|}{ IUGR (N=30) } \\
\hline \multirow{2}{*}{ CPR } & \multicolumn{2}{|c|}{ Low APGAR } & \multirow{2}{*}{\multicolumn{2}{|c|}{ Total }} \\
\hline & Present & Absent & & \\
\hline Low & $12(40.0 \%) \mathrm{TP}$ & $8(26.7 \%) \mathrm{FP}$ & & $\%)$ \\
\hline Normal & $2(6.7 \%) \mathrm{FN}$ & $8(26.7 \%) \mathrm{TN}$ & & \\
\hline Total & $14(46.7 \%)$ & $16(53.3 \%)$ & & $\%)$ \\
\hline Kappa $(95 \%$ CI $)$ & \multicolumn{2}{|c|}{$0.384(0.042-0.653)$} & $P$ & $<0.001^{*}$ \\
\hline \multicolumn{5}{|c|}{ Non-IUGR (N=30) } \\
\hline Low & $5(16.7 \%) \mathrm{TP}$ & $6(20 \%) \mathrm{FP}$ & & \\
\hline Normal & $1(3.3 \%) \mathrm{FN}$ & $18(60.0 \%) \mathrm{TN}$ & & \\
\hline Total & $6(20.0 \%)$ & $24(80.0 \%)$ & & $\%)$ \\
\hline Kappa $(95 \%$ CI $)$ & \multicolumn{2}{|c|}{$0.444(0.120-0.769)$} & $P$ & $<0.001^{*}$ \\
\hline
\end{tabular}

Percentages are from the total (30), TP: True positive, TN: True negative, FP: False positive, FN: False negative

Table 5: Agreement between low CPR and meconium aspiration

\begin{tabular}{|c|c|c|c|}
\hline \multicolumn{4}{|c|}{ IUGR (N=30) } \\
\hline \multirow{2}{*}{ CPR } & \multicolumn{2}{|c|}{ Meconium aspiration } & \multirow{2}{*}{ Total } \\
\hline & Present & Absent & \\
\hline Low & $13(43.3 \%) \mathrm{TP}$ & $7(23.3 \%) \mathrm{FP}$ & $20(66.7 .0 \%)$ \\
\hline Normal & $0(0.0 \%) \mathrm{FN}$ & $10(33.3 \%) \mathrm{TN}$ & $10(33.3 \%)$ \\
\hline Total & $13(43.3 \%)$ & $17(56.7 \%)$ & $30(100.0 \%)$ \\
\hline Kappa (95\% CI) & \multicolumn{2}{|c|}{$0.553(0.294-0.812)$} & $<0.001^{*}$ \\
\hline \multicolumn{4}{|c|}{ Non-IUGR (N=30) } \\
\hline Low & $4(13.3 \%) \mathrm{TP}$ & $7(23.3 \%) \mathrm{FP}$ & $11(36.7 \%)$ \\
\hline Normal & $1(3.3 \%) \mathrm{FN}$ & $18(60.0 \%) \mathrm{TN}$ & $19(63.3 \%)$ \\
\hline Total & $5(16.7 \%)$ & $25(83.3 \%)$ & $30(100.0 \%)$ \\
\hline Kappa $(95 \%$ CI) & \multicolumn{2}{|c|}{$0.351(0.025-0.678)$} & $<0.001^{*}$ \\
\hline
\end{tabular}

Percentages are from the total (30), TP: True positive, TN: True negative, FP: False positive, FN: False negative

Table 6: Agreement between low CPR and hypoxic ischemic encephalopathy

\begin{tabular}{lccc}
\hline \multicolumn{3}{c}{ IUGR (N=30) } \\
\hline CPR & Encephalopathy & Total \\
Low & Present & Absent & $20(66.7 .0 \%)$ \\
Normal & $6(20.0 \%) \mathrm{TP}$ & $14(46.7 \%) \mathrm{FP}$ & $10(33.3 \%)$ \\
Total & $0(0.0 \%) \mathrm{FN}$ & $10(33.3 \%) \mathrm{TN}$ & $30(100.0 \%)$ \\
Kappa (95\% CI) & $6(20.0 \%)$ & $24(80.0 \%)$ & 0.053 \\
& & $0.222(0.035-0.409)$ & $11(36.7 \%)$ \\
Low & Non-IUGR (N=30) & $19(63.3 \%)$ \\
Normal & $1(3.3 \%) \mathrm{TP}$ & $10(33.3 \%) \mathrm{FP}$ & $30(100.0 \%)$ \\
Total & $0(0.0 \%) \mathrm{FN}$ & $29(96.7 \%)$ & $P$ \\
Kappa (95\% CI) & $1(3.3 \%)$ & $0.351(0.025-0.678)$ & 0.181 \\
\hline
\end{tabular}

Percentages are from the total (30), TP: True positive, TN: True negative, FP: False positive, FN: False negative 
Table 7: Agreement between low CPR and NICU admission

\begin{tabular}{|c|c|c|c|c|}
\hline \multicolumn{5}{|c|}{ IUGR $(\mathrm{N}=30)$} \\
\hline \multirow{2}{*}{ CPR } & \multicolumn{2}{|c|}{ NICU admission } & \multirow{2}{*}{\multicolumn{2}{|c|}{ Total }} \\
\hline & Present & Absent & & \\
\hline Low & $15(50.0 \%) \mathrm{TP}$ & $5(16.7 \%) \mathrm{FP}$ & & $\%)$ \\
\hline Normal & $1(3.3 \%) \mathrm{FN}$ & $9(30.0 \%) \mathrm{TN}$ & & \\
\hline Total & $16(53.3 \%)$ & $14(46.7 \%)$ & & $\%)$ \\
\hline Kappa $(95 \%$ CI) & \multicolumn{2}{|c|}{$0.591(0.310-0.827)$} & $P$ & $<0.001 *$ \\
\hline \multicolumn{5}{|c|}{ Non-IUGR (N=30) } \\
\hline Low & $5(16.7 \%) \mathrm{TP}$ & $6(20.0 \%) \mathrm{FP}$ & & \\
\hline Normal & $2(6.6 \%) \mathrm{FN}$ & $17(56.7 \%) \mathrm{TN}$ & & \\
\hline Total & $7(23.3 \%)$ & $23(76.7 \%)$ & & $\%)$ \\
\hline Kappa $(95 \%$ CI $)$ & \multicolumn{2}{|c|}{$0.444(0.120-0.769)$} & $P$ & $<0.001 *$ \\
\hline
\end{tabular}

Percentages are from the total (30), TP: True positive, TN: True negative, FP: False positive, FN: False negative

Table 8: Agreement between low CPR and neonatal death

\begin{tabular}{|c|c|c|c|c|}
\hline \multicolumn{5}{|c|}{ IUGR $(\mathrm{N}=30)$} \\
\hline CDP & \multicolumn{2}{|c|}{ Neonatal death } & \multirow{2}{*}{\multicolumn{2}{|c|}{ Total }} \\
\hline CPR & Present & Absent & & \\
\hline Low & $3(10.0 \%) \mathrm{TP}$ & $17(56.7 \%) \mathrm{FP}$ & \multicolumn{2}{|c|}{$20(66.7 .0 \%)$} \\
\hline Normal & $0(0.0 \%) \mathrm{FN}$ & $10(33.3 \%) \mathrm{TN}$ & \multicolumn{2}{|c|}{$10(33.3 \%)$} \\
\hline Total & $3(10.0 \%)$ & $27(90.0 \%)$ & \multicolumn{2}{|c|}{$30(100.0 \%)$} \\
\hline Kappa (95\% CI) & \multicolumn{2}{|c|}{$0.105(0.000-0.230)$} & $P$ & 0.197 \\
\hline \multicolumn{5}{|c|}{ Non-IUGR (N=30) } \\
\hline Low & $1(3.3 \%) \mathrm{TP}$ & $10(33.3 \%) \mathrm{FP}$ & \multicolumn{2}{|c|}{$11(36.7 \%)$} \\
\hline Normal & $0(0.0 \%) \mathrm{FN}$ & $19(63.3 \%) \mathrm{TN}$ & \multicolumn{2}{|c|}{$19(63.3 \%)$} \\
\hline Total & $1(3.3 \%)$ & $29(96.7 \%)$ & \multicolumn{2}{|c|}{$30(100.0 \%)$} \\
\hline Kappa $(95 \%$ CI $)$ & \multicolumn{2}{|c|}{$0.112(0.000-0.319)$} & $P$ & 0.181 \\
\hline
\end{tabular}

Percentages are from the total (30), TP: True positive, TN: True negative, FP: False positive, FN: False negative

\section{DISCUSSION}

Predictability of gestation having the of adverse perinatal outcome development is a cornerstone obstetric challenges in every day practice. sonography could aid in detectability of those clinically challenging gestations e.g by growth restriction identification. On the other hand, fetal growth pattern solely couldn't detect all foetuses at risk and could easily miss those category that grow within normal ranges but are not approaching their maximum growth potential[ ${ }^{[4]}$.

Doppler flow indices of placental and fetal vessels when properly integrated and calculated could enhance the detectability of the detailed fetal hemodynamic status and placental perfusion pathologies that could affect the clinical prognosis of perinatal outcomes ${ }^{[14]}$.
The current research is a case control study that was held in Ain Shams university maternity hospital, during the period between March 2019 to October 2019. The aim was to evaluate the role of cerebro-placental ratio in predictability of perinatal outcome in high-risk category of pregnancies having intrauterine growth restriction.

This research recruited 60 high risk pregnant women categorized in to two research groups:

Group I: study group: 30 high risk pregnant women with IUGR.

Group II: control group: 30 high risk pregnant women without IUGR.

As regards the demographic research data of study subjects recruited, the mean age was $28.8 \pm 3.0$ years, the mean BMI was $27.2 \pm 2.4 \mathrm{Kg} / \mathrm{m} 2$, the mean gestational age 
was $35.7 \pm 1.5$ weeks and regarding the parity $70 \%$ of the women were PG and $30 \%$ were multipara.

The current study showed that among patients with IUGR, 20 (66.7.0\%) had low CPR, $10(33.3 \%)$ had normal CPR, while in patients without IUGR, $11(36.7 \%)$ had low CPR, 19 (63.3\%) had normal CPR. This shows that Low CPR was statistically significantly more frequent among IUGR research categorical group.

Alaa Ebrashy et al., also reported abnormal cerebroplacental ratio in $41.8 \%$ cases of preeclampsia without IUGR and $84.2 \%$ cases of preeclampsia with IUGR. Our study corresponds with this study ${ }^{[7]}$.

Najam et al., the sensitivity, specificity, positive predictive value (PPV), and negative predictive value (NPV) for detection of IUGR for umbilical artery Doppler were $48.15,80.67,53.06$, and $77.41 \%$, while that for MCA Doppler were 59.25, 88.89, 72.72, and $81.35 \%$. Forabnormal $\mathrm{C} / \mathrm{U}$ ratio, the values were $85.18,89.72$, $80.70 \%$, and 92.30 . So, CPR is most sensitive with higher PPV for detection of IUGR. Those research findings could be justified by the fact that cerebroplacental ratio analyses two different flow indices in an integrated manner that represents the fetoplacental vascular status in a more representive manner ${ }^{[15]}$.

Abnormal CPR has been associated with an increased risk of perinatal complications, especially in IUGR fetuses from PE pregnancy those findings are justified by the fact that placental vascular pathology causing hypoperfusion of the fetus necessitates the trigger of brain spaing effect casing the abnormalities in cerebroplacental ratio ${ }^{[16]}$.

CPR could be used to identify fetuses that have a higher risk of hemodynamic pathological changes before birth, CPR being considered as a marker of placental hypoperfusion and reduced placental functional reserve ${ }^{[9]}$.

There is reduced resistance within fetal circulation once the pregnancy progresses to term. However resistance within middle cerebral artery should remain higher than in the umbilical artery. Therefore, CPR should be greater than 1-1.1 in an uncomplicated pregnancy ${ }^{[17]}$.

The current study showed that among patients with IUGR, 19 (63.3\%) had CS for fetal distress, 14 (46.7\%) had neonate with low APGAR, $13(43.3 \%)$ had neonatal meconium aspiration, $6(20.0 \%)$ had neonatal Hypoxic ischemic Encephalopathy, $16(53.3 \%)$ had neonate need NICU admission, 3 (10.0\%) had neonatal death, 1 (3.3\%) had neonatal still birth, while in patients without IUGR, 11 (36.7\%) had CS for fetal distress, 6(20.0\%) had neonate with low APGAR, 5 (16.7\%) had neonatal meconium aspiration, $1(3.3 \%)$ had neonatal Hypoxic ischemic Encephalopathy, $7(23.3 \%)$ had neonate need NICU admission, 1 (3.3\%) had neonatal death, $0(0.0 \%)$ had neonatal still birth. This shows that Poor outcomes were more frequent among IUGR group; the differences were statistically signfciant except Hypoxic ischemic encephalopathy, death and still birth. Those research findings coud be justified by the fact that pregnancies affected by placental vascular hypo perfusion issues have lower capability to withstand normal hypoxic challenges that could arise during the physiological process of delivery ${ }^{[24]}$.

Abnormal CPR has been associated with an increased risk of perinatal complications, especially in IUGR fetuses from PE pregnancy ${ }^{[16]}$.

The current study showed that low CPR in IUGR group had $89.5 \%$ sensitivity, $72.7 \%$ Specificity, $83.3 \%$ Diagnostic accuracy, $60.0 \%$ positive predictive value and $80.0 \%$ negative predictive value to predict CS for fetal distress, while the low CPR in non-IUGR group had $72.7 \%$ sensitivity, $84.2 \%$ Specificity, $80.0 \%$ Diagnostic accuracy, $72.7 \%$ positive predictive value and $84.2 \%$ negative predictive value to predict CS for fetal distress. So, Low CPR had moderate diagnostic characteristics in predicting CS for fetal distress in study groups.

Also Eser et al. (2011), found statically significant positive correlation between abnormal CPR with fetal distress as $P$ value $=0.0008, P$ value $=0.004$, respectively, the results showed that $\mathrm{CP}$ ratio had $34.7 \%$ sensitivity and $96 \%$ specificity to detect Fetal distress, with positive predictive value $96 \%$ and negative predictive value $12 \%{ }^{[8]}$.

The current study showed that low CPR in IUGR group had $85.7 \%$ sensitivity, $50.0 \%$ Specificity, $66.7 \%$ Diagnostic accuracy, $60.0 \%$ positive predictive value and $80.0 \%$ negative predictive value to predict low APGAR, while the low CPR in non-IUGR group had $83.3 \%$ sensitivity, 75.0\% Specificity, 76.7\% Diagnostic accuracy, 45.5\% positive predictive value and $94.7 \%$ negative predictive value to predict low APGAR. So, Low CPR had moderate diagnostic characteristics in predicting low APGAR in study groups.

On the other hand Eser et al. (2011), in their cross sectional study that included 185 women, that studied the predictive value of $\mathrm{CP}$ ratio in prediction of neonatal outcomes in preeclampsia. showed no significant positive correlation between abnormal $\mathrm{CP}$ ratio and poor Apgar score, as $P$ value $=0.1$, showed no significant positive correlation between abnormal $\mathrm{CP}$ ratio and poor Apgar score, as $P$ value $=0.1$, The results showed that $\mathrm{CP}$ ratio had $42.8 \%$ sensitivity and $74.3 \%$ specificity, $9.7 \%$ positive predictive value and $94.1 \%$ negative predictive value, $9.7 \%$ positive predictive value and $94.1 \%$ negative predictive value to predict newborns with Apgar $5 \mathrm{~min}<7$, but didn't study APGAR $1 \mathrm{~min}^{[8]}$.

The current study showed that low CPR in IUGR group had $100.0 \%$ sensitivity, $58.8 \%$ Specificity, $76.7 \%$ Diagnostic accuracy, $65.0 \%$ positive predictive value and $100.0 \%$ negative predictive value to predict meconium aspiration, while the low CPR in non-IUGR group had $80.0 \%$ sensitivity, $72.0 \%$ Specificity, $73.3 \%$ Diagnostic accuracy, $36.4 \%$ positive predictive value and $94.7 \%$ 
negative predictive value to predict meconium aspiration. So, Low CPR had high sensitivity and low specificity in predicting meconium aspiration in study groups.

The current study showed that low CPR in IUGR group had $100.0 \%$ sensitivity, $41.7 \%$ Specificity, $53.3 \%$ Diagnostic accuracy, $30.0 \%$ positive predictive value and $100.0 \%$ negative predictive value to predict hypoxic ischemic encephalopathy, while the low CPR in nonIUGR group had $100.0 \%$ sensitivity, $65.5 \%$ Specificity, $66.7 \%$ Diagnostic accuracy, $9.1 \%$ positive predictive value and $100.0 \%$ negative predictive value to predict hypoxic ischemic encephalopathy. So, Low CPR had high sensitivity and low specificity in predicting hypoxic ischemic encephalopathy in study groups.

These results agreed with the results obtained by Eser et al. (2011), that show significant positive correlation between abnormal CPR with fetuses with NICU admission, as $P$ value $=0.0006, P$ value $=0.0009$, respectively, also Smitha et al. (2014) showed significant positive correlation as $P$ value $<0.001^{[8]}$.

Shahinaj et al. (2010), in their prospective observational study that included 738 singleton pregnancies, that studied The value of the $\mathrm{CP}$ ratio in the prediction of neonatal outcome in patient with preeclampsia, found positive correlation and statistically significant between abnormal $\mathrm{CP}$ ratio and Neonatal death as $P$ value $<.0001$, the result showed that $\mathrm{CP}$ ratio had $97.7 \%$ sensitivity and $66.0 \%$ specificity to predict Neonatal death, with positive predictive value $16.5 \%$ and negative predictive value $99.7 \%$.

The current study showed that low CPR in IUGR group had $100.0 \%$ sensitivity, $34.5 \%$ Specificity, $36.7 \%$ Diagnostic accuracy, $5.0 \%$ positive predictive value and $100.0 \%$ negative predictive value to predict still birth, while the low CPR in non-IUGR group had 63.3\% Specificity and $36.7 \%$ Diagnostic accuracy to predict still birth. So, Low CPR had high sensitivity and low specificity in predicting still birth in IUGR group.

A prior meta-analysis with the aim to evaluate usage of cerebroplacental ratio in predictability of adverse perinatal clinical outcome. The research data have revealed and displayed that abnormal cerebroplacental ratio could predict the requirement for operative mode of delivery necessary for foetal distress $[P$ value $<0.001]$, low $\mathrm{pH}[P$ value $=0.005]$ and low Apgar scoring $[P$ value $=0.10]$, foetal or neonatal death [relative risk $=2.49,95 \%$ confidence interval $=1.00-6.20]$, besides NICU admission $[P$ value $=0.14]$. The research team of investigators from the data analysis have revealed and displayed a statistically significant correlation as regards all outcomes except the low $\mathrm{pH}$. Those research findings show great similarity and harmony to the current research study findings ${ }^{[13]}$.

\section{CONCLUSION}

Cerebro -placental ratio has high predictive value of perinatal outcome in high risk pregnancy with intrauterine growth restriction.

\section{CONFLICT OF INTEREST}

There are no conflicts of interest.

\section{REFERENCES}

1. Albu AR, Anca AF, Horhoianu VV, Horhoianu IA Predictive factors for intrauterine growth restriction. Journal of medicine and life. 2014; 7(2):165.

2. American College of Obstetricians and Gynecologists. Diagnosis and management of preeclampsia and eclampsia: ACOG Practice Bulletin No. 33. Obstet Gynecol 2012; 99:159-167.

3. Baschat AA. Arterial and venous Doppler in the diagnosis and management of early onset fetal growth restriction. Early Hum Dev 2005; 81:877-87.

4. Buck-Louis GM, et al. Racial/ethnic standards for fetal growth: the NICHD fetal growth studies. Am J Obstet Gynecol 2015; 213(4):449.

5. Cosmi E, Fanelli T, Visentin S, Trevisanuto D, Zanardo $\mathrm{V}$. Consequences in infants that were intrauterine growth restricted. Journal of pregnancy. 2011; 2011.

6. Devaskar SU and Chu A. Intrauterine growth restriction: hungry for an answer. Physiology. 2016; 31(2):131-46.

7. Ebrashy O, Azmy O, Ibrahim M. Middle cerebral umbilical artery resistance index ratio as sensitive parameter for fetal well being and neonatal outcome in patients with the eclampsia. Case Control Study. Croat J Med J.2005; 46 (5): 826-831.

8. Eser A, Zulfikarolgue E, Eserdag S, Kihc S and Damsman N. predictive value of middle cerebral artery to uterine artery pulsatility index ratio in preeclampsia. Arch Gynecol. Obstet 2011; 284: 307-311.

9. Etchegaray A, Moren J, Ciammella R, Esteban M. Cerebroplacental ratio (CPR) at term as a marker of intrapartum placental reserve: results of a retrospective cohort. Ultrasound in Obstetrics and Gynecology. 2016; 48(1):57-57.

10. Gardosi J and Francis A. Adverse pregnancy outcome and association with small for gestational age birthweight by customized and population-based percentiles. Am J Obstet Gynecol 2009; 201:28.e1-8. 
11. Khanduri S, Chhabra S, Yadav S, Sabharwal T, Chaudhary M, Usmani T, Goyal A, Sharma H. Role of Color Doppler Flowmetry in Prediction of Intrauterine Growth Retardation in High-Risk Pregnancy. Cureus. 2017; 9(11).

12. McCowan LM, Figueras F, Anderson NH. Evidencebased national guidelines for the management of suspected fetal growth restriction: comparison, consensus, and controversy. American journal of obstetrics and gynecology. 2018; 218(2):S855-68.

13. Moreta D, Samuel V, Guy D, Eslick RB. Re-evaluating the role of cerebroplacental ratio in predicting adverse perinatal outcome European Journal of Obstetrics and Gynecology and Reproductive Biology 2019; 242: 17-28.

14. Muresan D, Rotar IC, Stamatian F. The usefulness of fetal Doppler evaluation in early versus late onset intrauterine growth restriction. Review of the literature. Med Ultrason 2016; 18(1):103-9.

15. Najam R, Gupta S and Shalini O. Predictive Value of Cerebroplacental Ratio in Detection of Perinatal Outcome in High-Risk Pregnancies; The Journal of Obstetrics and Gynecology of India 2018; 66(4):244-247.

16. Novac MV, Moldoveanu A, Tudorache Ş, et al. Utility of Cerebroplacental Ratio in IUGR Fetuses from Pregnancy with Preeclampsia in Prediction the Risk for Perinatal Complications. Curr Health Sci J. 2017;43(3):231-235.

17. Ropacka-Lesiak M, Korbelak T, Swider-Musielak J, Breborowicz G. Cerebroplacental ratio in prediction of adverse perinatal outcome and fetal heart rate disturbances in uncomplicated pregnancy at 40 weeks and beyond. Arch Med Sci. 2015;11(1):142-148.
18. Schlaudecker EP, Munoz FM, Bardají A, Boghossian NS, Khalil A, Mousa H, Nesin M, Nisar MI, Pool V, Spiegel HM, Tapia MD. Small for gestational age: Case definition and guidelines for data collection, analysis, and presentation of maternal immunisation safety data. Vaccine. 2017;35(48):6518-28.

19. Scifres CM, Stamilio D, Macones GA, Odibo AO. Predicting perinatal mortality in preterm intrauterine growth restriction. American journal of perinatology. 2009; 26(10):723-8.

20. Shahinaj R, Manoku N, Enriketa Kroi Eand lir Tasha. The value of the middle cerebral to umbilical artery Doppler ratio in the prediction of neonatal outcome in patient with preeclampsia and gestational hypertension. Journal of Prenatal Medicine 2010; 4 (2): 17-21.

21. Sharma D, Shastri S, Sharma P. Intrauterine growth restriction: antenatal and postnatal aspects. Clinical Medicine Insights: Pediatrics. 2016 Jan;10:CMPed-S40070.

22. Smitha K, Sowmya K and Malathi T. Study of Doppler waveforms in pregnancy induced hypertension and its correlation with perinatal outcome. Int J Reprod Contracept Obstet Gynecol. 2014; 3(2):428-433.

23. Soma-Pillay P, Catherine NP, Tolppanen H, Mebazaa A, Tolppanen H, Mebazaa A. Physiological changes in pregnancy. Cardiovascular journal of Africa. 2016; 27(2):89.

24. Twomey S, Flatley C, Kumar S. The association between a low cerebro- umbilical ratio at 30-34 weeks gestation, increased intrapartum operative intervention and adverse perinatal outcomes. Eur J Obstet Gynecol Reprod Biol 2016; 203:89-93. 